\title{
Structural and optical properties of Cr doped AIN thin films before and after ions implantation
}

Asmat Ullah Asmat ( $\square$ asmatullahphy@gmail.com )

Harbin Engineering University https://orcid.org/0000-0001-5538-5241

Muhammad Usman

National Centre for Physics

Altaf Hussain Shar

Harbin Engineering University

\section{Research Article}

Keywords: AIN:Cr, Irradiation, XRD, RBS, FTIR

Posted Date: July 15th, 2021

DOl: https://doi.org/10.21203/rs.3.rs-703467/v1

License: (c) (1) This work is licensed under a Creative Commons Attribution 4.0 International License. Read Full License 


\section{Abstract}

Thin films of Chromium ( $\mathrm{Cr}$ ) doped Aluminum Nitride (AIN) have been prepared by radio frequency magnetron sputtering technique at room temperature and pressure conditions in a Nitrogen $(\mathrm{N})$ atmosphere. One of the samples was left as-deposited and the other was irradiated with proton at a fluence of $1 \times 10^{14}$ ions $/ \mathrm{cm}^{2}$ at a dose of $335 \mathrm{keV}$. The impact of ions doping on the structural and optical characteristics were investigated systematically. RBS and XRD were utilized to study the stoichiometry, thickness and structural information of the thin films respectively. FTIR was utilized to understand the bonding chemistry of the specimens. We concluded that the optical properties of the thin films have been changed selectively with irradiation leaving by structural unaltered.

\section{Introduction}

Aluminum Nitride thin films semiconductor is providing fascinating properties in the field of optoelectronics and optics due to its unique bandgap (6.2 eV), high thermal and electrical characteristics. It is also considering a useful semiconductor host in order to enhance the luminescence depending upon the excitation in the EM spectrum lying in the visible, infrared and ultraviolet range. The rare earth elements when doped into these high bandgap semiconductors finds variety in diverse applications such as lasing, displays, biomedical and electronic applications [3-6]. The $4 \mathrm{f}$ orbitals of rare earth elements are shielded by the $4 \mathrm{~d}$ and $5 \mathrm{p}$ orbitals electrons which upon excitation is producing the EM spectrum containing infrared, visible and ultraviolet range [7]. The diameter of the atom of $\mathrm{Cr}$ is comparably higher so this mismatch perturb the crystallinity of AIN whereas AIN can be found in the structure of wurtzite, zincblende and rocksalt. This mismatch has role in the easy growth of thin films due to elimination of grain boundaries at low temperature growth $[8,9]$. The goal of this doping is the achievement of more efficient phosphor materials and/or in the development of photonic devices.

Irradiation of ions has been found a great tool in tuning the desired materials characteristics such as magnetic, electrical, piezoelectric, electronic, structural and mechanical characteristics [10-13].

In this manuscript we have investigated influence of the implanted ions into matrix of $\mathrm{Cr}$ doped AIN thin films on the structure, dimension and optical properties. We concluded that $\mathrm{Cr}$ doped AIN specimens remained unaffected from the structural standpoint while the optical properties have undergone changes.

\section{Synthesis And Irradiation On Samples}

The single crystalline Silicon (Si) wafer was used as the substrate of the thin films which was either cut along (100) or (111) plane. Different plane results different impact on the film's growth. The synthesis was carried out using RF magnetron sputtering method. The sputtering system was maintained by varying power ranged from 100 to $200 \mathrm{~W}$. An ultrahigh vacuum pump was connected to the system to maintain a vacuum less than $3 \times 10^{-5}$ Torr at room temperature. The Nitrogen $(\mathrm{N})$ was used as the 
sputtering gas while the target disk is Aluminum (Al). Figure. 1 shows the schematic representation of the deposition of the samples.

The sputtered $\mathrm{N}$ ions interact with the Al due to chemical affinity between them and deposit over there. The sputtering of $\mathrm{Cr}^{+3}$ is also taking place in parallel to Nitrogen's and the $\mathrm{Cr}^{+3}$ ions replace the Al by having substitutional sites [1]. After the deposition the samples were annealed thermally at a temperature of $900{ }^{\circ} \mathrm{C}$ in order to remove the porosity and homogeneous. The deposited and annealed sample was then cut into two halves and the one was left as grown and the other half was irradiated with protons in a room temperature and pressure at a dose of $1 \times 10^{14} \mathrm{ion} / \mathrm{cm}^{2}$ with $335 \mathrm{keV}$ using pelletron tandem accelerator facility at national center for Physics, Islamabad, Pakistan.

\section{Results And Discussion}

Rutherford Backscattering Spectroscopy (RBS) is an analytical method to study the composition of the thin films. This technique do the quantification without the use of reference standards and is a non destructive. The RBS analysis is the bombardment of high-energy (MeV) $\mathrm{He}^{2+}$ ions (i.e. alpha particles) directed unto the surface of the specimens and the backscattered particle's yield and distribution of energy is measured at a given specific angle. The information about the backscattering cross section for each element can be obtain from literature review and is known for each sample so it is possible to get the knowledge of quantitative compositional depth profile from the obtained spectrum of RBS. The beam energy used was $2.085 \mathrm{MeV}$ while the beam current was set to $29 \mathrm{nA}$ with charge of $20 \mu \mathrm{C}$ was used in the data collection. The bounced ions after striking the surface were detected by $\mathrm{Si}$ detector and the angle was set to $170^{\circ}$. The figure. 1 is depicting the simulation and experimental yields of the relative concentration of elements present in the sample against the channel.

SIMNRA (SIMulation of Nuclear Reaction Analysis) is a windows program using for the simulation of charged particle energy spectra and gamma ray yields gamma-ray yields for ion beam analysis with incident ions from about $100 \mathrm{keV}$ to many $\mathrm{MeV}$. The films parameters were evaluated using RBS analysis when fitted with SIMNRA. The used input parameters set in the SIMNRA calculations in order to do the fitting of experimental and simulated curves were scattering angles $\theta$ (deg), calibration offset (keV), exit angle $\beta$ (deg), detector resolution (keV) and energy per channel (keV/ch) are given by respectively $170^{\circ}$, $35.41,10^{0}, 20.99$ and 1.15 . The films composition obtained from the RBS spectroscopy are shown in table. 1.

Table 1

Percent Composition and thickness of the film

\begin{tabular}{|lllll|}
\hline Al & $\mathbf{N}$ & $\mathbf{0}$ & $\mathbf{C r}$ & Thickness $(\mathrm{nm})$ \\
\hline 34.2 & 17.6 & 45.9 & 2.1 & 400 \\
\hline
\end{tabular}


In order to study the chemical bonds existing in the specimens and to detect the optical vibrations we conducted the Fourier Transform Infrared (FTIR) Spectroscopy using the FT/IR-6600 type setup. This spectroscopy informs us that each molecule within the samples has an associated frequency whereby reflecting or transmitting the rest of the existing frequencies which could carry the information about the strength and nature of the bond [14]. These vibration motions consist of vibrational transitions with distinct energies. The goal of this experiment was to find the amount of light that to be transmitted. Figure. 3 is contrasting the transmittance in percent value against the wavenumber for the thin film $\mathrm{Cr}$ doped AIN thin films.

The result concluded that the transmittance has been lowered with the irradiation and the number and positions of the peaks have been altered. The transmittance has been lowered down by almost 3 orders of magnitude. The existence of peaks in the range from $2270-2420 \mathrm{~cm}^{-1}$ represents $\mathrm{CO}_{2}$, which is due to the spectrometer path and is not from composition of the films $[15,16]$ while the peaks at $1067 \mathrm{~cm}^{-1}$ represents Si-O-Si $[17,18]$.

$X$ Ray Diffraction (XRD) is an analytical technique conducted in this study for the identification of phase of a crystalline material as well for the information about the dimension of the unit cell. The structural information of the films was obtained by using XRD. The source of $x$-rays was copper with wavelength of $1.5418 \mathrm{~A}^{0}$.

The peaks at (003), (012) and (111) are the corresponded crystallographic axes of Si. No change in the peaks has been found with the irradiation other than slight increase in the intensity which is informing us about the enhanced crystalline behavior while the peaks at (200), (110) and (101) correspond to AIN. The sharpness of the peaks is almost unaffected with the physical treatment.

\section{Conclusions}

Thin films of $\mathrm{Cr}$ doped $\mathrm{AIN}$ has been prepared with reactive magnetron sputtering technique in a room temperature and pressure. After annealing at $900{ }^{\circ} \mathrm{C}$ the film was irradiated with protons using $1 \times 10^{14}$ ions $/ \mathrm{cm}^{2}$ dose with an impinging energy of $335 \mathrm{keV}$. The structural and optical properties were investigated and found that the irradiated has significantly altered the optical properties by decreasing the transmittance while the structure of the films remained unaffected with selective irradiations parameters which prove that the irradiation can selectively modify desired characteristics of the semiconductor.

\section{Declarations}

\section{Acknowledgment}

This work was financially supported by national center for Physics, Islamabad, Pakistan 


\section{References}

1. Asmat Ullah M, Usman W, Qingyu I, Ahmad RY, Khosa, Muhammad Maqbool "Response of structural and optical properties against proton irradiation in AIN:Tm thin films"

https://doi.org/10.1016/j.radphyschem.2020.109234

2. Mayer M (2014) Improved physics in SIMNRA 7. Nucl Instrum Methods B 332(1 August):176-180

3. Maqbool M et al (2007) Appl Phys Lett 91:193511

4. Chuah LS et al (2010) Int J Mod Phys B 24:1129

5. Maqbool M (2009) et al., Nanoscale Res Lett 4 (:748)

6. Hassan N, Hussain Z, Naeem M, Shah IA, Husnain G, Ahmad I, Ullah Z (2017) Influence of ions beam irradiation on the structural, magnetic and electrical characteristics of Ho doped AlN thin films. Surf Rev Lett 24, No.(2 0:1750021 “"', )

7. E. Michael et al., Properties of Advanced Semiconductor Materials: GaN, AIN, InN, BN, SiC, SiGe (John Wiley and Sons, New York, 2001)

8. Kim YH (2015) et al., Thin Solid Films 576 (:61 )

9. Iriarte GF (2010) et al., Mater Res Bull 45 (:1039)

10. Summers GP, Burke EA, Shapiro P, Messenger SR, Walters RJ (1993) Damage Co-relations in semiconductors exposed to gamma, electron and proton radiation. Tran nuc sci $40(: 6-14$,

11. Muhammad Usman A, Hallen A, Nazir, Ion implantation induced nitrogen defects in GaN, J Phys Appl Phys 48 (2015) 455107, https://doi.org/10.1088/ 0022-3727/48/45/455107

12. Shakil, Khan (2014) et al., Structural characteristics of Nip-implanted AIN thin film. Surf Topogr Metrol Prop 2 (:35007. https://doi.org/10.1088/2051-672X/2/3/ 035007, )

13. Asmat Ullah Q, Wang IA, Muhammad Usman "Irradiation effects on Nd and W doped Aluminum Nitride thin films" https://doi.org/10.1016/j.physb.2020.412086

14. Fox M (2001) Optical Properties of Solids. Oxford University press, UK, pp. 5-32

15. Sankaramahalingam Angappana RA, Jeneafer A, Visuvasama LJ, Berchmans (2013) Synthesis of AIN - presence and absence of additive". Estonian J Eng 19(3):239-249 0

16. Seki K, Xu X, Okabe H, Frye JM, Halpern JB (1992) Room-temperature growth of AIN thin films" by laser ablation. Appl Phys Lett 60 (:2234-2236, )

\section{Figures}




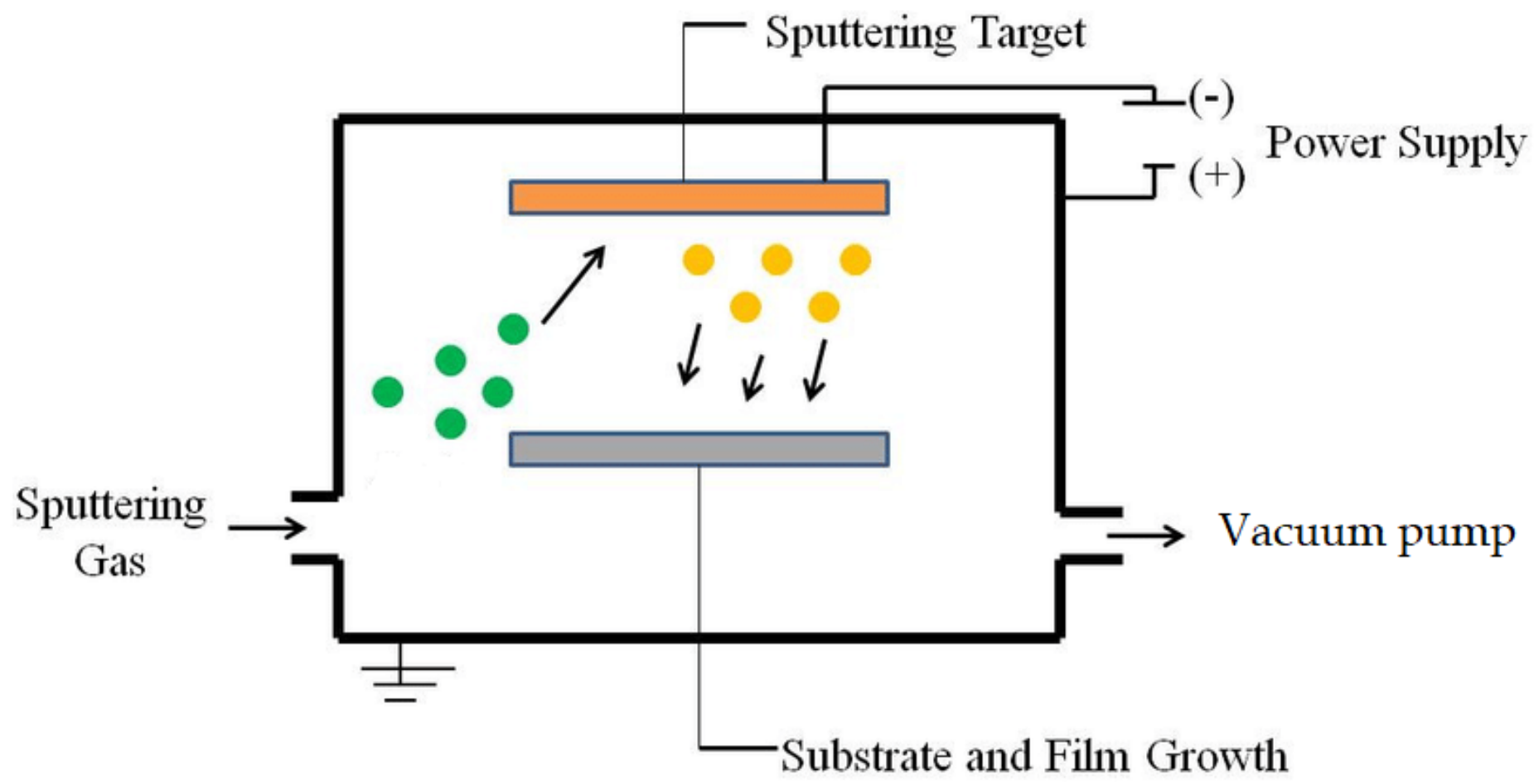

Figure 1

Schematic of sputtering deposition process for the thin film's growth 


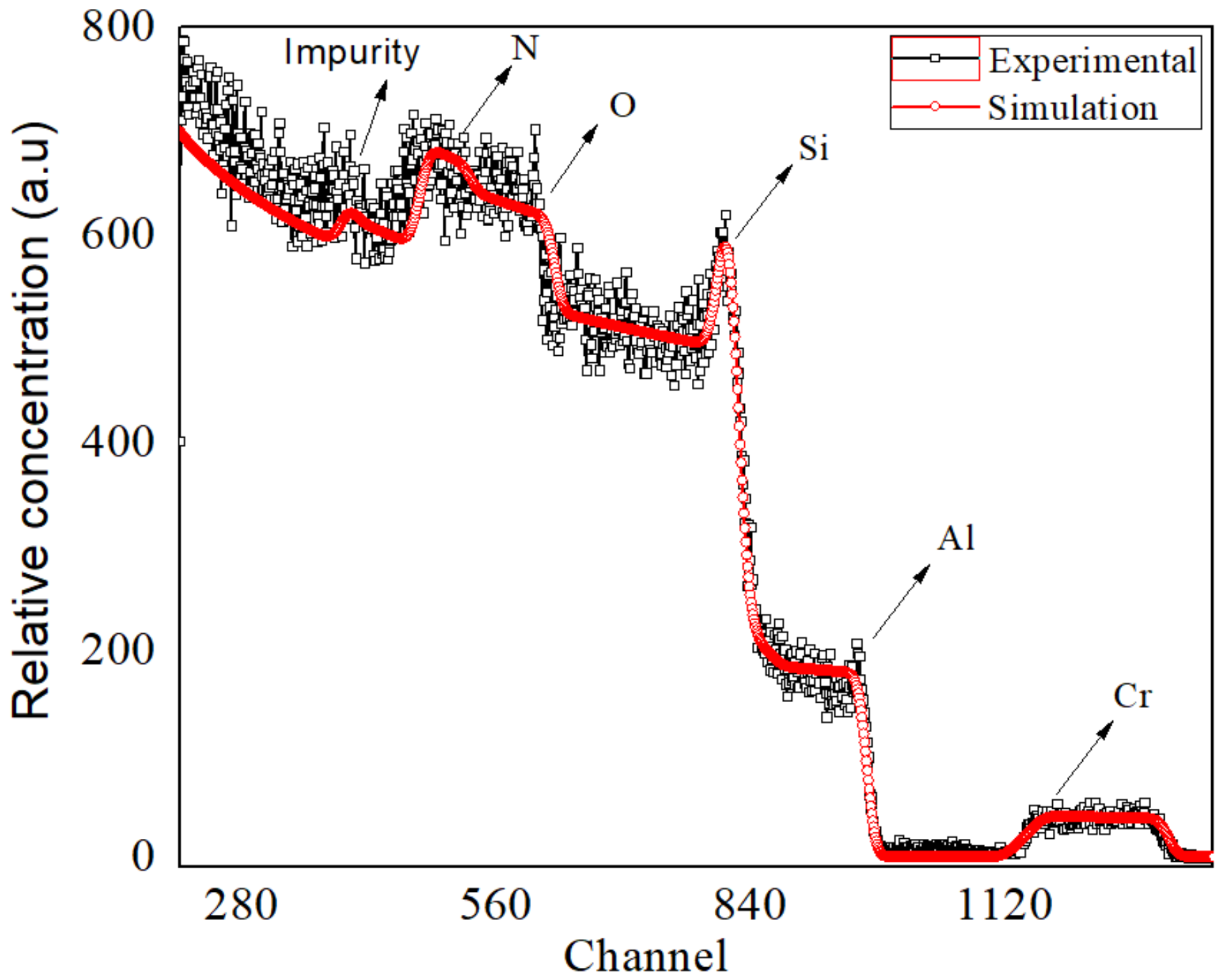

Figure 2

RBS spectroscopy of AIN:Cr conducted at $2 \mathrm{MeV} \mathrm{He++} \mathrm{bombardment}$ 


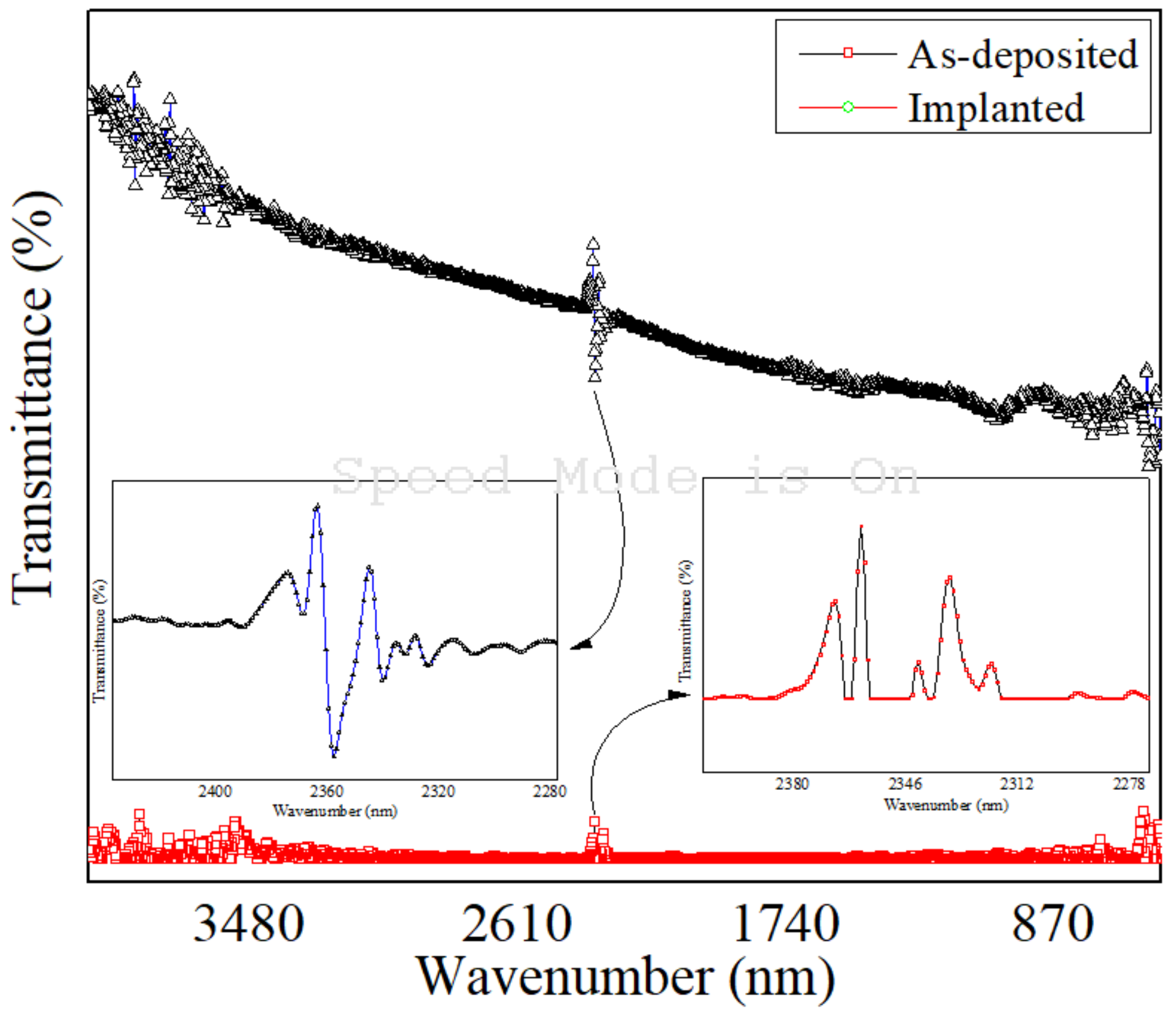

Figure 3

FTIR spectroscopy of AIN:Cr before and after the proton irradiation conducted at room temperature 


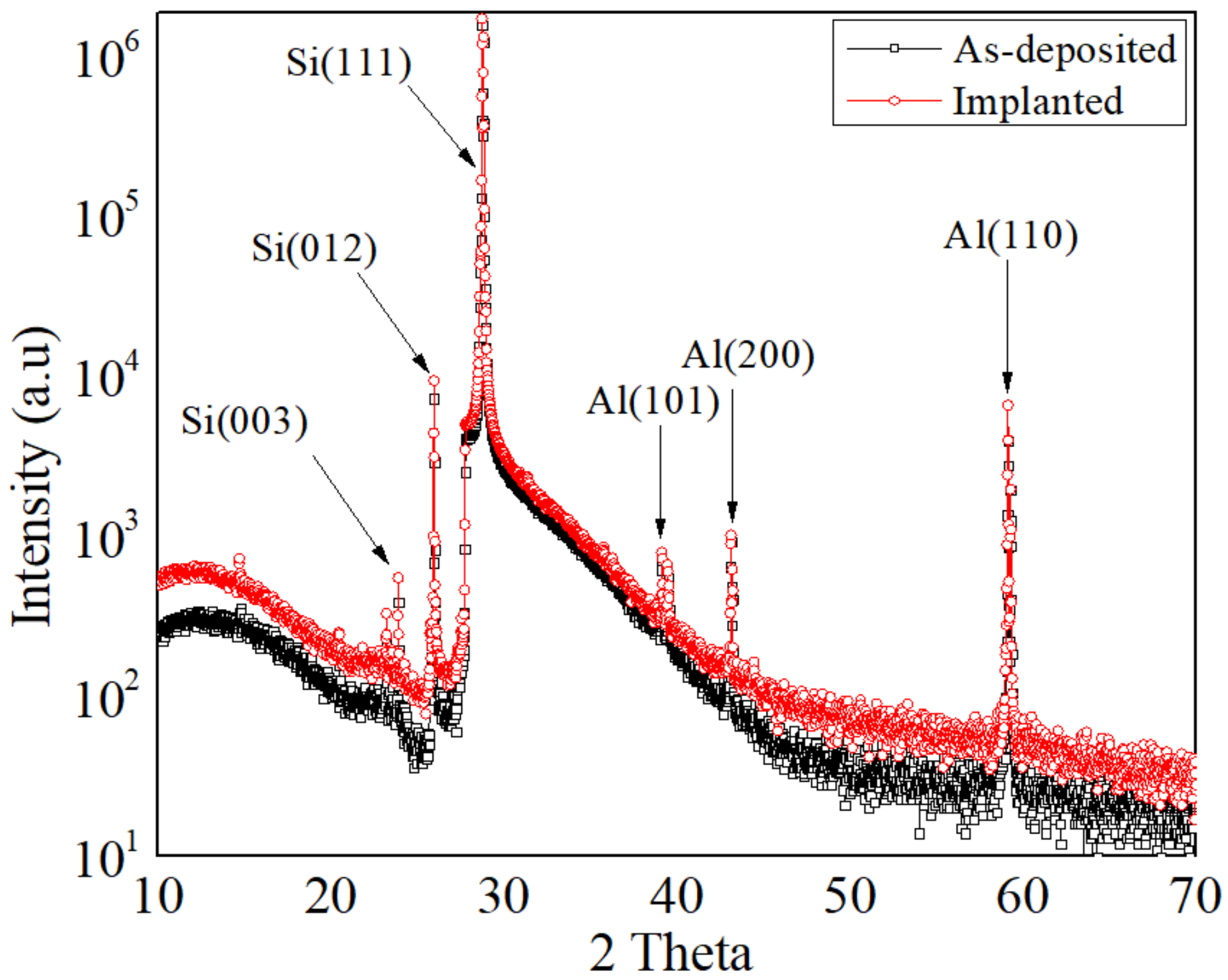

Figure 4

XRD pattern of AIN:Cr before and after the irradiation 